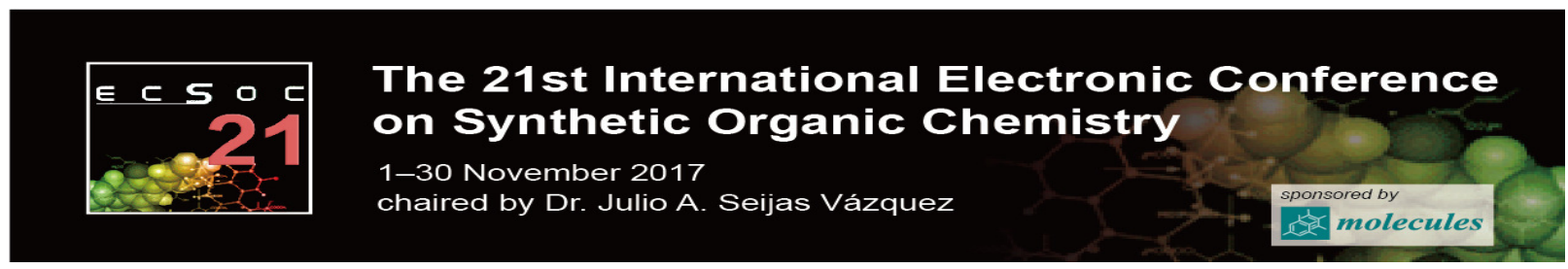

http://sciforum.net/conferencelecsoc-21

\title{
Basic GO-Imidazolium ionic liquid as a recoverable nanocatalyst for the
}

\section{synthesis of pyrrole derivatives}

\author{
Hamid Reza Esmaili Zand, ${ }^{\text {a }}$ Hossein Ghafuri*a \\ ${ }^{a}$ Catalysts and Organic Synthesis Research Laboratory, Department of Chemistry, Iran \\ University of Science and Technology, Tehran, Iran \\ *E-mail: Ghafuri@iust.ac.ir
}

\begin{abstract}
Ionic liquid with basic properties is a significant category in the wide-ranging ionic liquid field. Ionic liquids are mostly made of ions and ion pairs. They are known as powerful solvents which have potential applications. Since the 1999 review by Welton which is about room temperature ionic liquids, ionic liquids have grown as a motivating research area in the last 15 years. Some ionic liquids are liquid at room temperature include some nitrogen-based compounds. Among them, imidazolium-based ionic liquids are the most important ones. In imidazole-based ionic liquids, their physical properties like melting point and acidity-basicity could be adjusted by changing the alkyl substituents and ion pairs. Most of room temperature ionic liquids involve bulky organic cations such as 1-alkyl-3-methylimidazolium, 1-alkylpyridinium and ammonium ion. To have heterogeneous nanocatalysts using ionic liquids, it was studied that carbonaceous substrates with high surface area like graphene oxide could be the best choice.
\end{abstract}


To this purpose and to have heterogeneous basic nanocatalyst with high surface area and significant catalytic activity, basic GO-Imidazolium ionic liquid was synthesized in this research. Here, N-methyl imidazole was used as cation, HCO3- as anion and GO as solid support to form basic ionic liquid nanocatalyst and its catalytic ability was used in synthesis of pyrroles as a significant N-heterocycle compound with important biological and pharmacological activity.

Keywords: Basic ionic liquid, recoverable nanocatalyst, pyrroles

\section{Introduction}

Due to ionic liquid's exclusive properties such as a wide liquid range, good solvating ability, tunable polarity, high thermal stability, negligible vapor pressure, and ease of recyclability, there has been considerable interest in the use of ionic liquids as an environmentally benign reaction media [1]. polymerization [2], hydrogenation [3], regioselective alkylation [4], Friedel-Crafts reactions [5], dimerization of alkenes [6], Diels-Alder reactions [7], Michael reactions [8], crosscoupling reactions [9] and some enzymic reactions [10] are the examples of some organic reactions which can be carried out in ionic liquids. Basic ionic liquids showed various advantages such as the catalytic efficiency and recycling of the ionic liquid than the combination of inorganic base and ionic liquid for some base-catalyzed processes $[11,12]$ and have also aroused unprecedented interest.

Basic GO-Imidazolium ionic liquid has been successfully applied for the first time to catalyze the synthesis of pyrroles. However, the ability of an ionic liquid as a clean catalyst and reagent has not been explored to any great extent although it is of much importance in the context of the green synthesis $[13,14]$. 
In our search for simple catalytic route to pyrrole derivatives, herein we first reported an environmentally benign methodology for this reaction.

\section{Experimental section}

\subsection{General}

Reagents and chemicals were all purchased from Aldrich, Merck and Fluka. Melting points were measured with an Electrothermal 9100 apparatus and are uncorrected. Spectra of FT-IR were recorded by the method of $\mathrm{KBr}$ pellet on a Shimadzu IR-470 spectrometer.

\subsection{General synthesis of pyrrole derivatives}

Basic GO-Imidazolium ionic liquid (50 mg) was added to a stirred solution of $2 \mathrm{mmol}$ amine and 2 mmol 2,5-hexandione round-bottom flask. The mixture was stirred at room temperature for the desired time. After completion reaction, the solid catalyst was filtered and washed. The pure products could be obtained by evaporated under vacuum.

\section{Results and discussion}

The synthesis reaction of pyrrole can be done by combination of benzaldehyde with various diones (table 1). The optimized conditions for the reaction was using $0.05 \mathrm{~g}$ of catalyst in EtOH and at room temperature. 
Scheme 1. Synthesis of pyrroles in optimal conditions.

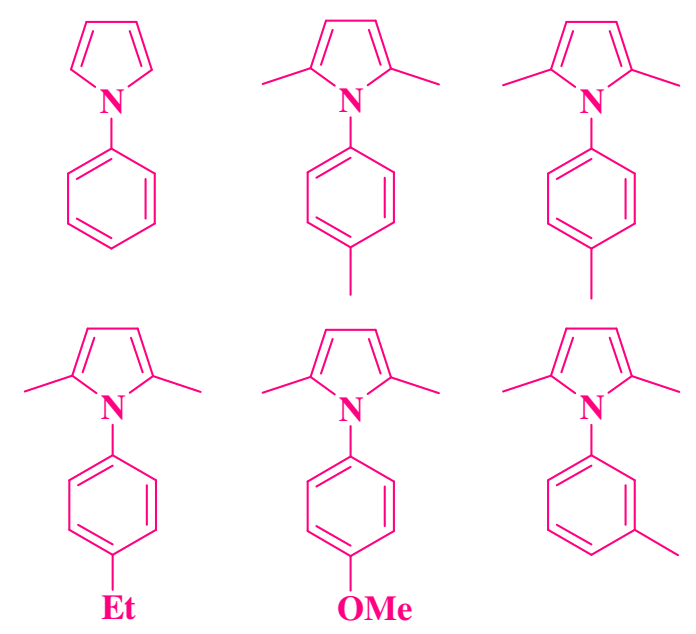

\subsection{Spectral data of 2,5-dimethyl-1-p-tolyl-1H-pyrrole}

IR (KBr): (vmax): 3084, 3076, 2992, 2981, 2764, 1946, 1589, $1463 \mathrm{~cm}^{-1} ;{ }^{1} \mathrm{H} \mathrm{NMR}\left(\mathrm{CDCl}_{3}, 300\right.$ $M H z): \delta 2.13(s, 6 H), 2.30(s, 3 H) 5.76(s, 2 H), 7.01(m, 2 H), 7.41-7.65(m, 3 H) ;{ }^{13} C N M R(100$ $M H z, C D C l 3): 12.54,24.3,104.53,126.85,128.01,128.71,128.91,139.78$.

\section{Conclusion}

In conclusion, a highly efficient basic GO-Imidazolium ionic liquid catalyzed the Pyrrole synthesis in mild condition reactions. The process was simple and generated a diverse range of pyrroles in excellent yields. it is notable that the use of recyclable basic ionic liquid makes this procedure quite simple and environmentally benign.

\section{Acknowledgements}

The authors gratefully acknowledge the partial support from the Research Council of the Iran University of Science and Technology. 


\section{References}

[1] Welton, T. Chem. Rev. 1999, 99, 2071.

[2] Abdul-sada, A. A. K.; Ambler, P. W.; Hodgson, P. K. G.; Seddon, K. R.; Stewart, N. J. World Pat. WO 9521871, 1995.

[3] Chauvin, Y.; Mussman, L.; Olivier, H. Angew. Chem., Int. Ed. Engl. 1995, 38, 3097.

[4] Badri, M.; Brunet, J. J.; Perron, R. Tetrahedron Lett. 1992, 33, 4435.

[5] Boon, J. A.; Levisky, J. A.; Pflug, J. L.; Wilkes, J. S. J. Org. Chem. 1986, 54, 480.

[6] Ellis, B.; Keim, W.; Wasserscheid, P. Chem. Commun. 1999, 337.

[7] Earle, M. J.; McCormac, P. B.; Seddon, K. R. Green Chem. 1999, 1, 23.

[8] Corey, E. J.; Zhang, F. Y. Org. Lett. 2000, 2, 1097.

[9] Kaufmann, D. E.; Nouroozian, M.; Henze, H. Synlett 1996, 1091.

[10] Schoefer, S. H.; Kaftzik, N.; Wasserscheid, P.; Kragl, U. Chem. Commun. 2001, 425.

[11] Formentin, P.; Garcia, H.; Leyva, A. J. Mol. Catal. A: Chem. 2004, 214, 137.

[12] Ranu, B. C.; Banerjee, S. Org. Lett. 2005, 7, 3049.

[13] Xu, J.-M.; Liu, B.-K.; Wu, W.-B.; Qian, C.; Wu, Q.; Lin, X.-F. J. Org. Chem. 2006, 71, 3991.

[14] Harjani, J. R.; Nara, S. J.; Salunkhe, M. M. Tetrahedron Lett. 2002, 43, 1127. 